\title{
Activated c-SRC in ductal carcinoma in situ correlates with high tumour grade, high proliferation and HER2 positivity
}

\author{
GR Wilson ${ }^{1,4}$, A Cramer ${ }^{3}$, A Welman ${ }^{4}$, F Knox ${ }^{2}$, R Swindell ${ }^{3}$, H Kawakatsu ${ }^{5}$, RB Clarke ${ }^{6}$, C Dive ${ }^{4}$ \\ and NJ Bundred*,I
}

'Department of Academic Surgery, Research and Education Building 2nd floor, South Manchester University Hospital, Southmoor Road, Wythenshawe, Manchester M23 9LT, UK; ${ }^{2}$ Department of Pathology, South Manchester University Hospital, Southmoor Road, Wythenshawe, Manchester M23 9LT, UK; ${ }^{3}$ South Manchester University and Christie Hospital NHS Trusts, Manchester, UK; ${ }^{4}$ Cellular \& Molecular Pharmacology, Cancer Research-UK, Paterson Institute for Cancer Research, Manchester, UK; ${ }^{5}$ Lung Biology Centre, University of California, San Fransisco, USA; ${ }^{6}$ Breast Biology Group, Division of Cancer Studies, University of Manchester, Paterson Institute for Cancer Research, Manchester, UK

\begin{abstract}
Overexpression and/or activity of c-Src non-receptor tyrosine kinase is associated with progression of several human epithelial cancers including breast cancer. c-Src activity in 'pure' ductal carcinoma in situ (DCIS) was measured to assess whether this predicts recurrence and/or correlates with HER2 expression and other clinical parameters. Activated c-Src levels were evaluated in DCIS biopsies from 129 women, with median follow-up at 60 months. High levels of activated c-Src correlated with HER2 positivity, high tumour grade, comedo necrosis and elevated epithelial proliferation. In univariate analysis, high activated c-Src level associated with lower recurrence-free survival at 5 years $(P=0.01 \mathrm{I})$. Thus, high $c$-Src activity may identify a subset of DCIS with high risk of recurrence or progression to invasive cancer where therapeutics targeting c-Src may benefit this patient subset.

British Journal of Cancer (2006) 95, 1410-14|4. doi:10.1038/sj.bjc.6603444 www.bjcancer.com

Published online 24 October 2006

(c) 2006 Cancer Research UK
\end{abstract}

Keywords: c-Src; breast cancer; DCIS; immunohistochemistry; HER2

Overexpression and/or increased activity of c-Src non-receptor tyrosine kinase is associated with development of many human carcinomas including breast cancer (Irby and Yeatman, 2000) where it is elevated early in disease progression (Verbeek et al, 1996). c-Src overexpression is also associated with breast tumour metastasis (Guy et al, 1994; Muthuswamy and Muller, 1994), especially to bone (Myoui et al, 2003). The molecular events driving c-Src activation in breast cancers are unclear although this can occur downstream of activated receptor tyrosine kinases (RTKs) including HER2/erbB2 (Muthuswamy et al, 1994; BelschesJablonski et al, 2001). Overexpression of constitutively activated Src drives oncogenic processes that could contribute to latter stages of carcinogenesis and most prominently, increased motility and invasion (Frame, 2004). Few studies have examined subtypes of breast cancer with respect to c-Src activity and as Src-targeted therapies are entering the clinic, data are now required to determine which patient groups (disease type and stage) might optimally benefit.

There is a $12-20 \%$ recurrence rate at 10 years following breast conserving surgery and adjuvant radiotherapy for DCIS and up to half of recurrences are invasive carcinomas (Fisher et al, 1999). c-Src binds to, and can be activated by HER2 and appears to play a critical role in HER2-mediated breast cancer invasion and

*Correspondence: Professor NJ Bundred; E-mail: nigel.j.bundred@manchester.ac.uk

Received 8 June 2006; revised 25 September 2006; accepted 27 September 2006; published online 24 October 2006 metastasis (Tan et al, 2005). Here, we investigated whether high levels of active c-Src are associated with clinico-pathological factors including HER2 status and early recurrence of DCIS.

\section{MATERIALS AND METHODS}

\section{Patients and tissues}

One hundred and twenty-nine cases of women diagnosed with 'pure' DCIS (median patient age 55 years, median follow-up 60 months) were selected from the University Hospital of South Manchester database. Thirty-five cases recurred within 5 years and 94 cases had not recurred. All specimens were formalin-fixed and paraffin-embedded. Patient age, histological nuclear grade, tumour size, follow-up and recurrence times were retrieved from our unit database (Barnes et al, 2005). Ethical approval was from South Manchester University Hospital Ethics Board.

\section{Immunohistochemistry and Western blotting}

mAb Clone 28 recognizes an epitope adjacent to Tyrosine 530 in the $\mathrm{C}$ terminal regulatory domain of activated c-Src (Kawakatsu et al, 1996). Sections ( $4 \mu \mathrm{m})$ of tissue were processed as previously described (Boland et al, 2004) before incubation with Clone 28 $(1: 800)$ for $60 \mathrm{~min}$. Antibody binding was detected using antimouse DakoCytomation Envision + System HRP kit (Dako, UK). Incubation time with $3.3^{\prime}$-diaminobenzidine and substratechromogen depended on positive control staining intensity 
( $\sim 8 \mathrm{~min}$ ). Sections were counterstained as previously described (Boland et al, 2004). Mouse IgG2a $\kappa(1: 50)$ provided a negative control. HCT116 cells engineered to inducibly express wild-type (wt) or constitutively activated c-Src (c-SrcY527F) (Welman et al, 2005) were used as positive control pellets, processed and analysed identically to clinical specimens. Induction of wt c-Src (or c-SrcY527F) was confirmed by Western blotting using pAb rabbit anti-Src (pY418, Biosource, UK no. 44-660). Activated c-Src staining was also investigated in a MDA MB231 breast cancer cell tumour xenograft, chosen as this cell line exhibits high levels of activated c-Src measured by Western blotting (Ito et al, 2002). Activated c-Src expression was also evaluated in sections of normal breast ductal epithelium by IHC. Inter-assay variation was assessed in high and low scoring sections form one batch in the subsequent batches. All slides were examined and scored by two independent observers, one blinded to clinical outcome. Total cellular activated c-Src staining intensity was scored using a scale of 0 (negative), 1 (low), 2 (moderate) and 3 (high). To qualify as 3, 2 and 1 dark staining of $100,>70$ or $<70 \%$ of tumour cells had to be observed. The IHC evaluation of ER status, HER2 expression and Ki67 levels was as previously described (Boland et al, 2004). To assess intraand inter-observer variability, 60 sections were selected at random and scored twice by the same observer (G.W., 4 of 60 scores differed) and scored independently by two observers (G.W. and A.C, 3 of 60 cases scores differed). Slides that differed were re-analysed in conference and a representative score agreed.

\section{RESULTS}

\section{Activated c-Src staining in different breast tissue}

Initially, the ability of clone 28 to recognize activated c-Src using the IHC protocols above was confirmed using HCT116 cell pellet controls. When high levels of wt c-Src (Figure 1A(iii), (or c-Src $\mathrm{Y} 527 \mathrm{~F}$, not shown), were induced, staining was strongly positive compared to uninduced cells (Figure 1Aii). Controls with mouse IgG did not stain (Figure 1A(i)). Western blotting confirmed that wt c-Src was activated in HCT116 when induced (Figure 1 inset) thus these data together confirmed that clone 28 can reliably probe active $\mathrm{c}-\mathrm{Src}$ by IHC. Figure $1 \mathrm{~A}(\mathrm{v})$ shows positive staining with clone 28 staining of the MDA MB231 breast cancer cell xenograft consistent with the reported high levels of this breast cell line (Ito et al, 2002).

Activated c-Src staining was detected in all DCIS specimens with higher levels in DCIS compared with normal breast tissue, and correlated positively with tumour nuclear grade (Figure 1B(i-iv)). Low-level-activated c-Src was detected in $12 \%$ normal breast tissue sections. Of 129 DCIS tumours analysed 19\% tumours expressed low activated c-Src levels, $41 \%$ expressed moderate and $40 \%$ expressed high levels. Moderate immuno-reactivity was often observed in lymphocytes surrounding DCIS.

\section{Activated c-Src expression $v s$ clinico-pathological variables}

Table 1 summarises the relationship between expression of total cellular activated c-Src and clinico-pathological factors. High levels of activated c-Src in DCIS was associated with HER2 positivity $(P<0.0005)$, high tumour nuclear grade $(P<0.0005)$, the presence of comedo necrosis $(P=0.001)$ and high Ki67 scores $(P=0.025)$, but not with ER status $(P=0.973)$, tumour size $(P=0.403)$ and EGFR/HER1 expression $(P=0.507)$.

\section{Activated c-Src and recurrence-free survival}

Univariate analysis Activated $\mathrm{c}$-Src level was a significant predictor of DCIS recurrence at 5 years $(P=0.011)$. Patients with DCIS expressing higher levels of activated $\mathrm{c}$-Src had a poorer cumulative 5 year disease-free survival compared to those with low
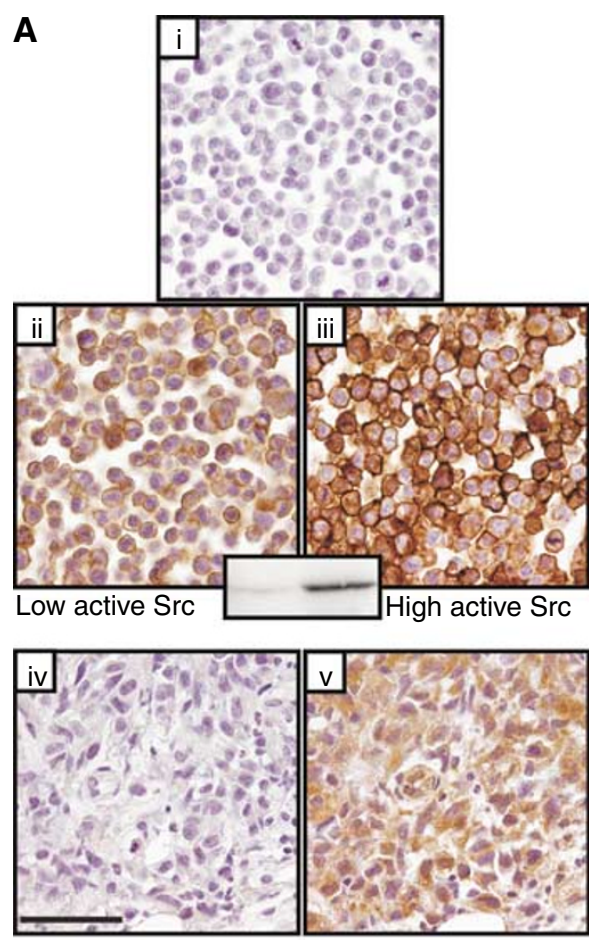

B

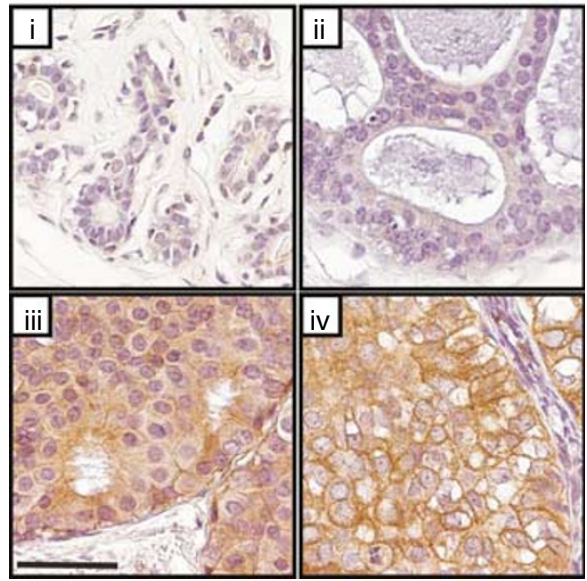

Figure I $(\mathbf{A i}-\mathbf{v})$ Experimental controls for Clone 28 immunohistochemistry of activated c-Src. Pellets of untreated or doxycycline treated HCTI I 6 colon cancer cells that express a doxycycline-inducible, wild-type (wt) c-Src. High levels of activated c-Src were detected in doxycyline treated HCTI 16 colon cancer cells harbouring the doxycycline-inducible wt c-Src (Aiii). Detectable but lower levels of active c-Src were detected in these HCTII6 cells in the absence of doxycyline treatment (Aii). Induction of wt c-Src was independently confirmed by western blotting using an anti $\mathrm{Src}\left[\mathrm{pY}{ }^{418}\right]$ antibody specific for autophosphorylated active cSrc (Figure IA insert. No significant immunoreactivity was detected in HCTI I 6 cells analysed by mouse IgG2a, which served as a negative control (Ai). Figure IA(iv) shows a section of a MDA MB23I tumour xenograft negative control (IgG2A) and $\mid A(\mathbf{v})$ shows clone positive staining with Clone 28 in this xenograft indicative of high levels of activated c-Src. (Bi-iv) Immunohistochemistry for activated c-Src using clone 28 in normal breast epithelium $(\mathbf{B i})$, and DCIS tumours (Bii-iv). Low levels of activated c-Src in $\mathbf{B}$ (ii), moderate levels in $\mathbf{B}(\mathbf{i i i})$ and high levels in $\mathbf{B}(\mathbf{i v})$. A higher level of activated c-Src was detected in DCIS tumours compared with normal breast. There was a positive correlation between the level of activated c-Src and tumour nuclear grade $(P<0.0005)$. The scale bar equals $50 \mu \mathrm{m}$. 
Table I Correlations between the total cellular expression levels of activated c-Src (low, moderate, and high) and clinicopathologic factors in 129 DCIS tumours

\begin{tabular}{|c|c|c|c|c|}
\hline \multirow[b]{2}{*}{ Clinical Factor } & \multicolumn{3}{|c|}{ Level of activated c-Src } & \multirow[b]{2}{*}{$P$-value } \\
\hline & Low $(n=25)$ & $\begin{array}{l}\text { Moderate } \\
(n=53)\end{array}$ & High $(n=5 I)$ & \\
\hline \multicolumn{5}{|c|}{ HERI status $(n=43)$} \\
\hline Positive $(\geqslant 2)$ & 5 & 10 & 15 & \\
\hline Negative $(<2)$ & 3 & 6 & 4 & $0.507^{\mathrm{a}}$ \\
\hline \multicolumn{5}{|c|}{ HER2 status $(n=125)$} \\
\hline Positive $(\geqslant 2)$ & 10 & 29 & 42 & \\
\hline Negative $(<2)$ & 15 & 21 & 8 & $<0.0005^{\mathrm{a}}$ \\
\hline \multicolumn{5}{|l|}{$E R$ status $(n=126)$} \\
\hline Positive & 16 & 34 & 32 & \\
\hline Negative & 8 & 18 & 18 & $0.973^{\mathrm{a}}$ \\
\hline \multicolumn{5}{|l|}{$\operatorname{Ki67}(n=125)$} \\
\hline Median & 11.2 & 11.0 & 13.7 & \\
\hline Range & $2.5-31.4$ & $1.3-61.1$ & $2.6-46.0$ & $0.025^{b}$ \\
\hline \multicolumn{5}{|c|}{ Tumour nuclear grade $(n=128)$} \\
\hline Low & 5 & 4 & 0 & \\
\hline Intermediate & 11 & 21 & 6 & \\
\hline High & 9 & 27 & 45 & $<0.0005^{a}$ \\
\hline \multicolumn{5}{|c|}{ Histological type $(n=125)$} \\
\hline Comedo & 3 & 11 & 11 & \\
\hline Mixed comedo & 6 & 22 & 32 & \\
\hline Non-comedo & 14 & 20 & 6 & $0.001^{\mathrm{a}}$ \\
\hline \multicolumn{5}{|c|}{ Tumour size $(\mathrm{mm})(n=123)$} \\
\hline Median & 17.5 & 13.5 & 20 & \\
\hline Range & $5-40$ & $4-43$ & $4-75$ & $0.403^{b}$ \\
\hline
\end{tabular}

High expression levels of activated c-Src in DCIS correlated with HER2 positivity, high tumour grade, the presence of comedo necrosis and higher epithelial proliferative status but not with tumour size, ER status and EGFR expression. Statistical analysis: ${ }^{a} \chi^{2}$ test. ${ }^{b}$ Kruskall - Wallis test.

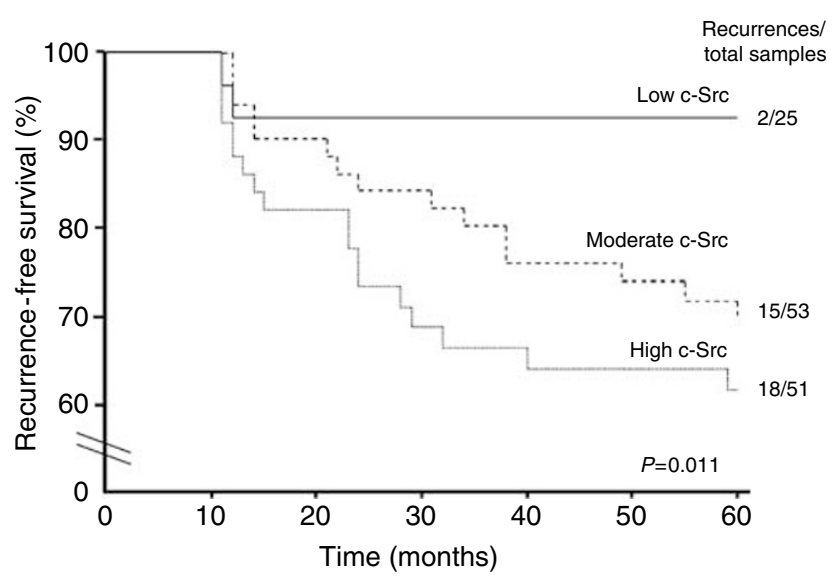

Figure 2 In univariate analysis, Kaplain-Meier plot of cumulative local recurrence (DCIS and invasive breast carcinoma) for 129 patients with DCIS. Low-activated c-Src expressing DCIS tumours had a significant disease-free survival advantage over DCIS expressing moderate to high levels of activated c-Src.

levels (61.7 vs $92 \%, P=0.011$, Figure 2). Of the 35 DCIS tumours that recurred by 5 years, $6 \%$ expressed low levels of activated c-Src, $43 \%$ expressed moderate levels and $51 \%$ expressed high levels
Table 2 Univariate analysis of predictors of DCIS recurrence at 5 years using the log rank test

\begin{tabular}{|c|c|c|c|}
\hline Clinical factor & $\begin{array}{l}\text { Non-recurrent } \\
\quad(n=94)\end{array}$ & $\begin{array}{l}\text { Recurrent } \\
(n=35)\end{array}$ & $P$-value \\
\hline \multicolumn{4}{|c|}{ HER2 status (\%) $(n=125)$} \\
\hline Positive $(\geqslant 2)$ & $52(57.8)$ & $29(82.9)$ & \\
\hline Negative $(<2)$ & $38(42.2)$ & $6(17.1)$ & 0.01 \\
\hline \multicolumn{4}{|c|}{ Ki67 score (\%) $(n=125)$} \\
\hline$<12.2$ (median) & $52(57.8)$ & $10(28.6)$ & \\
\hline$\geqslant 12.2$ & $38(42.2)$ & $25(71.4)$ & 0.006 \\
\hline \multicolumn{4}{|c|}{ Activated c-Src (\%) $(n=129)$} \\
\hline Low & $23(24.5)$ & $2(5.7)$ & \\
\hline Moderate & $38(40.4)$ & $15(42.9)$ & \\
\hline High & $33(35.1)$ & $18(51.4)$ & 0.011 \\
\hline \multicolumn{4}{|c|}{ ER status $(\%)(n=126)$} \\
\hline Positive & $61(67.0)$ & $21(60.0)$ & \\
\hline Negative & $30(33.0)$ & $14(40.0)$ & 0.524 \\
\hline \multicolumn{4}{|c|}{ Tumour grade $(\%)(n=128)$} \\
\hline Low & $9(9.7)$ & $0(0.0)$ & \\
\hline Intermediate & $34(36.5)$ & $4(11.4)$ & \\
\hline High & $50(53.8)$ & $31(88.6)$ & 0.0003 \\
\hline \multicolumn{4}{|l|}{ Margins (\%) $(n=129)$} \\
\hline Involved $(<1 \mathrm{~mm})$ & $78(83.0)$ & $19(54.3)$ & \\
\hline Clear $(\geqslant 1 \mathrm{~mm})$ & $16(17.0)$ & $16(45.7)$ & 0.001 \\
\hline \multicolumn{4}{|c|}{ Patient age (years) $(n=129)(\%)$} \\
\hline$<50$ & $6(6.4)$ & $8(22.9)$ & \\
\hline$\geqslant 50$ & $88(93.6)$ & $27(77.1)$ & 0.019 \\
\hline
\end{tabular}

HER2 positivity, high epithelial proliferation, high levels of total cellular activated c-Src, high tumour nuclear grade, involved margins and young age at presentation were associated with a higher disease recurrence at 5 years.

Table 3 Independent predictors of DCIS recurrence in the patient group $(n=129)$, carried out using Cox proportional hazards regression analysis

\begin{tabular}{lccccc}
\hline Clinical factor & B & s.e. & Exp (B) & $\mathbf{9 5 \%}$ Cl for Exp (B) & P-value \\
\hline $\begin{array}{l}\text { Margin status } \\
\quad \text { Involved }(<1 \mathrm{~mm})\end{array}$ & 0.967 & 0.344 & 2.63 & $1.34-5.17$ & 0.005 \\
$\begin{array}{l}\text { Patient age } \\
\quad<50 \text { years }\end{array}$ & 1.53 & 0.414 & 4.64 & $2.06-10.46$ & $<0.0005$ \\
$\begin{array}{l}\text { Tumour nuclear grade } \\
\quad \text { High }\end{array}$ & 1.751 & 0.536 & 5.76 & $2.01-16.47$ & 0.001 \\
\hline
\end{tabular}

(Figure 2). Other factors including HER2 status, Ki67, tumour nuclear grade, margin status and patient age were also significant predictors of disease recurrence (Table 2).

Multivariate analysis Higher tumour nuclear grade, involved margins $(<1 \mathrm{~mm})$ and higher patient age $(\geqslant 50$ years) were independently associated with an increased risk of recurrence at 5 years (Table 3 ). Activated c-Src level was not a significant independent predictor of recurrence-free survival.

\section{Activated c-Src $v s$ recurrence pathology}

Of the 35 women with recurrent DCIS, $37 \%$ had invasive recurrences and $63 \%$ had DCIS recurrence. The median recurrence time for invasive carcinoma was 24 months, compared with 13 months for DCIS recurrences. There was no significant difference between the level of activated c-Src and onset of DCIS recurrences 
(score low c-Src, 11 months; moderate, 12 months; high, 11 months). Of the 13 primary DCIS tumours that recurred as invasive carcinomas, $46 \%$ were moderate and $54 \%$ were high scores for activated c-Src. Of the 22 primary tumours that recurred as DCIS, $9 \%$ scored low $41 \%$ scored moderate and $50 \%$ scored high for activated c-Src.

\section{DISCUSSION}

Available data on activated c-Src levels in breast cancer relates mainly to invasive breast carcinoma. Our data combined with that of others (Ito et al, 2002; Diaz et al, 2006; Madan et al, 2006; Planas-Silva et al, 2006), suggests that compared to normal breast epithelium, increased levels of activated c-Src are present in DCIS and invasive breast carcinoma. Activated c-Src levels correlated with HER2 expression, a higher tumour nuclear grade, the presence of comedo necrosis, and higher epithelial proliferative status. Furthermore, high expression of activated c-Src correlated with significantly lower recurrence-free survival at 5 years. Consistent with the physical interactions between HER2 and c-Src, we observed a significant association between HER2 expression and activated c-Src. These data also resonate with function testing studies where overexpression of HER2 in transgenic mouse systems (Muthuswamy et al, 1994) and mammary epithelial cells (Sheffield, 1998) that resulted in c-Src activation. HER2 activation in human breast cancer cells increased c-Src protein level by driving its synthesis and/or stabilisation (Tan et al, 2005). However, highly expressed activated c-Src was also seen in HER2 negative DCIS thus HER2 independent pathways for activation must exist.

Although HER2 can activate c-Src, it is unclear if c-Src promotes proliferation. Discrepancies exist between activated c-Src levels and proliferation in invasive breast carcinoma (Ito et al, 2002). Here, activated c-Src levels positively correlated with increased cell proliferation but we cannot ascribe causality and there was no significant correlation between activated c-Src and tumour size.

Although numerous studies have demonstrated elevated c-Src activity in breast carcinoma (Jacobs and Rubsamen, 1983; Ottenhoff-Kalff et al, 1992; Verbeek et al, 1996), there have been discrepancies between tumour histological grade and activated cSrc (Ito et al, 2002). Here, levels of activated c-Src in DCIS strongly

\section{REFERENCES}

Barnes NL, Khavari S, Boland GP, Cramer A, Knox WF, Bundred NJ (2005) Absence of HER4 expression predicts recurrence of ductal carcinoma in situ of the breast. Clin Cancer Res 11: 2163-2168

Belsches-Jablonski AP, Biscardi JS, Peavy DR, Tice DA, Romney DA, Parsons SJ (2001) Src family kinases and HER2 interactions in human breast cancer cell growth and survival. Oncogene 20: 1465-1475

Boland GP, Butt IS, Prasad R, Knox WF, Bundred NJ (2004) COX-2 expression is associated with an aggressive phenotype in ductal carcinoma in situ. Br J Cancer 90: 423-429

Diaz N, Minton S, Cox C, Bowman T, Gritsko T, Garcia R, Eweis I, Wloch M, Livingston S, Seijo E, Cantor A, Lee JH, Beam CA, Sullivan D, Jove R, Muro-Cacho CA (2006) Activation of stat3 in primary tumors from high-risk breast cancer patients is associated with elevated levels of activated SRC and survivin expression. Clin Cancer Res 12: $20-28$

Fisher ER, Dignam J, Tan-Chiu E, Costantino J, Fisher B, Paik S, Wolmark N (1999) Pathologic findings from the National Surgical Adjuvant Breast Project (NSABP) eight-year update of Protocol B-17: intraductal carcinoma. Cancer 86: 429-438

Frame MC (2002) Src in cancer: deregulation and consequences for cell behaviour. Biochim Biophys Acta 1602: 114- 130

Frame MC (2004) Newest findings on the oldest oncogene; how activated src does it. I Cell Sci 117: 989-998 correlated with tumour nuclear grade (Figure 1B (ii-iv)) and comedo necrosis.

Elevated c-Src activity leads to inhibition of matrix metalloproteinases (Noritake et al, 1999), disruption of cell-cell adhesions (Yeatman, 2004) and enhances the migratory potential of tumour cells (Frame, 2002). We report here, that moderate to high levels of activated c-Src in DCIS recurred as invasive breast carcinomas and that increased activated c-Src level was a significant, but not independent predictor of disease recurrence.

In conclusion, we detected elevated activated c-Src in HER2 positive, high nuclear grade, DCIS tumours with a higher proliferative index and comedo necrosis. Our data support the hypothesis that in DCIS, c-Src may play a role in tumour proliferation, development of higher grade lesions and may facilitate progression to invasive recurrences and as such represent tractable drug targets in both DCIS and invasive breast carcinomas. HER2 overexpression in invasive breast cancer is associated with an aggressive phenotype, resistance to hormonal therapy and poor survival (e.g. Slamon et al, 1987). Only a third of HER2overpressing metastatic breast cancers respond to Trastuzumab (Herceptin), a function blocking anti-HER2 antibody (Vogel et al, 2002). c-Src targeted drugs are entering early clinical trials, and it is possible in appropriate patient cohorts that c-Src targeted drugs will be of benefit and that combination therapy with a c-Src inhibitor plus Herceptin may be superior to Herceptin alone.

\section{ACKNOWLEDGEMENTS}

We thank Miss Nicola Barnes, the Department of Academic Surgery, and the surgical staff from the South Manchester University and The Christie Hospital NHS Trust, Manchester, UK. We also thank $\mathrm{Mr} M$ Seenath, Dr Catharine West and Mr Garry Ashton for their assistance with the immunohistochemical staining and analysis of activated c-Src. We dedicate this paper to Dr H Kawakatsu (Lung Biology Centre, University of California, San Fransisco, USA \& Co-founder A-Cube Inc.) who died during this study.

\section{Additional Support}

GW, British Journal of Surgery Bursary Award.

RBC \& CD, Cancer Research-UK
Guy CT, Muthuswamy SK, Cardiff RD, Soriano P, Muller WJ (1994) Activation of the c-Src tyrosine kinase is required for the induction of mammary tumors in transgenic mice. Genes Dev 8: 23-32

Irby RB, Yeatman TJ (2000) Role of Src expression and activation in human cancer. Oncogene 19: $5636-5642$

Ito Y, Kawakatsu H, Takeda T, Tani N, Kawaguchi N, Noguchi S, Sakai T, Matsuura N (2002) Activation of c-Src is inversely correlated with biological aggressiveness of breast carcinoma. Breast Cancer Res Treat 76: $261-267$

Jacobs C, Rubsamen H (1983) Expression of pp60c-src protein kinase in adult and fetal human tissue: high activities in some sarcomas and mammary carcinomas. Cancer Res 43: 1696-1702

Kawakatsu H, Sakai T, Takagaki Y, Shinoda Y, Saito M, Owada MK, Yano J (1996) A new monoclonal antibody which selectively recognizes the active form of Src tyrosine kinase. J Biol Chem 271: 5680-5685

Madan R, Smolkin MB, Cocker R, Fayyad R, Oktay MH (2006) Focal adhesion proteins as markers of malignant transformation and prognostic indicators in breast carcinoma. Hum Pathol 37: 9-15

Muthuswamy SK, Muller WJ (1994) Activation of the Src family of tyrosine kinases in mammary tumorigenesis. Adv Cancer Res 64: 111-123

Muthuswamy SK, Siegel PM, Dankort DL, Webster MA, Muller WJ (1994) Mammary tumors expressing the neu proto-oncogene possess elevated c-Src tyrosine kinase activity. Mol Cell Biol 14: 735-743 
Myoui A, Nishimura R, Williams PJ, Hiraga T, Tamura D, Michigami T, Mundy GR, Yoneda T (2003) C-SRC tyrosine kinase activity is associated with tumor colonization in bone and lung in an animal model of human breast cancer metastasis. Cancer Res 63: 5028-5033

Noritake H, Miyamori H, Goto C, Seiki M, Sato H (1999) Overexpression of tissue inhibitor of matrix metalloproteinases-1 (TIMP-1) in metastatic MDCK cells transformed by v-src. Clin Exp Metastasis 17: 105-110

Ottenhoff-Kalff AE, Rijksen G, van Beurden EA, Hennipman A, Michels AA, Staal GE (1992) Characterization of protein tyrosine kinases from human breast cancer: involvement of the c-src oncogene product. Cancer Res 52: $4773-4778$

Planas-Silva MD, Bruggeman RD, Grenko RT, Stanley Smith J (2006) Role of c-Src and focal adhesion kinase in progression and metastasis of estrogen receptor-positive breast cancer. Biochem Biophys Res Commun 341: $73-81$

Sheffield LG (1998) C-Src activation by ErbB2 leads to attachmentindependent growth of human breast epithelial cells. Biochem Biophys Res Commun 250: 27-31

Slamon DJ, Clark GM, Wong SG, Levin WJ, Ullrich A, McGuire WL (1987) Human breast cancer: correlation of relapse and survival

with amplification of the HER-2/neu oncogene. Science 235: $177-182$

Tan M, Li P, Klos KS, Lu J, Lan KH, Nagata Y, Fang D, Jing T, Yu D (2005) ErbB2 promotes Src synthesis and stability: novel mechanisms of Src activation that confer breast cancer metastasis. Cancer Res 65: $1858-$ 1867

Verbeek BS, Vroom TM, Adriaansen-Slot SS, Ottenhoff-Kalff AE, Geertzema JG, Hennipman A, Rijksen G (1996) c-Src protein expression is increased in human breast cancer. An immunohistochemical and biochemical analysis. J Pathol 180: 383-388

Vogel CL, Cobleigh MA, Tripathy D, Gutheil JC, Harris LN, Fehrenbacher L, Slamon DJ, Murphy M, Novotny WF, Burchmore M, Shak S, Stewart SJ, Press M (2002) Efficacy and safety of trastuzumab as a single agent in first-line treatment of HER2-overexpressing metastatic breast cancer. J Clin Oncol 20: 719-726

Welman A, Cawthorne C, Barraclough J, Smith N, Griffiths GJ, Cowen RL, Williams JC, Stratford IJ, Dive C (2005) Construction and characterization of multiple human colon cancer cell lines for inducibly regulated gene expression. J Cell Biochem 94: 1148-1162

Yeatman TJ (2004) A renaissance for SRC. Nat Rev Cancer 4: 470-480 